\title{
Intuitive Factor Space Based on Perception Computing and Application in Criminal Investigation Reasoning
}

\author{
Weidong TAO, Ping $\mathrm{He}^{\star}$ \\ Department of Information, Liaoning Police College, China \\ lnpolice@126.com \\ *Corresponding author
}

\section{Keywords: Factor space; intuition characteristic; factors discovery; fuzzy reasoning}

\begin{abstract}
The purpose of this paper is to explore a kind of rational method according with the target selection of human perceptions behaviors: fuzzy perceptions of intuition. Based on intuitive factor space of the human mind for non-optimum perceptions and optimum perceptions, the paper analyses the formation and variation of fuzzy perceptions from the factor neural networks model of intuition process, and present the conception of trust fuzzy sets, then propose that the rational standards of intuition selection is an effective control of intuitive process under intuition index of factor perception (IIFP) conditions, finally design the trust fuzzy computing model. The studies of this paper introduce a new method for in determination fuzzy computing model based on intuitive factor space, and perfect the content of data mapping and intuition inversion.
\end{abstract}

\section{Introduction}

Ever since nearly half a century, the optimization theory has undoubtedly contributed extensively every branch of science and technology. It is because of its wide use that people find out it is far from actual requirements. People wonder whether ideal model analysis can solve real problems. Furthermore, it is very hard to build up a mathematic model for many of the actual complicated problems. Especially when the system is uncertain, man can only limply build up the model, but can hardly get its solution. Although there are a lot of approximate methods and theories of solving, they are far from satisfaction. In fact, there is acceptable (optimum attributes) and unacceptable (non-optimum attributes) aspect when the decision is made, that is, some attributes are both optimum and non-optimum, at the same time, there exist satisfactory results, as well as unsatisfactory ones, et al. But to some extent, the optimum and non-optimum can be judged of perception and measurement of relationship value. That is to say, people always exert the optimum attributes in the light of different decision condition, and knows more about how to overcome non-optimum attributes.

The original contribution of this paper relies in the modeling of structural information and rules using the opposite value set and perception degree, and the fusion of such pieces of cooperative perception in order to improve the optimization under uncertainty, to discuss a method for trusted optimum analysis based on cooperation of perception order and value order.

The paper is structured as follows: Section 2 presents the computational theory of factor perceptions, which is the basis for this work. Section 3 presents the application in criminal investigation reasoning. Section 4 draws a conclusion and further work.

\section{Basic Concepts and Method}

Factor Space Based on Perception. It is known that human brain, which can obtain process and evaluate factor, is a computing system of fuzzy perceptions processing. This computing system is constructed along with the experiences of processing and extents to use knowledge, and can be effectively discriminated to fuzzy information. Then what is human brain computing model of factor perception? In fact, computational model of factor perceptions is inspired by the remarkable human capability to perform a wide variety of physical and mental tasks, including recognition tasks, 
without any measurements and any computations. Recognition and perception are closely related. An essential difference between measurement and perception is that in general, measurements are crisp, while factor perceptions are fuzzy [1]. In existing theories, perceptions are converted into measurements, but such conversions in many cases, are infeasible, unrealistic or counterproductive. An alternative, suggested by the computing model of factor perception, is to convert perceptions into propositions expressed in a natural language.

An intuitive factor space can be represented by an information table where each row represents, for instance $f=\left\{f_{1}, f_{2}, \cdots, f_{n}\right\}$. Every column represents an attribute that can be measured for each intuitive factor; it can also be supplied by a human expert. The table is called a factor space. More formally, it is a pair $\mathbb{R}=(f, A)$ where $f$ is a non-empty finite set of factor called the universe and $A$ is a non-empty finite set of attributes such that $a: f \rightarrow V_{a}$ for every $a \in A$. The set $V_{a}$ is called the value set of $a$. If $V_{a}$ is not include null values, $\mathbb{R}$ is called a complete factor space, else $\mathbb{R}$ is called an incomplete factor space. For example, all voters take part in an election, there are three conditions in votes: assent, opposability, neutrality, then the space is complete factor space; if a part of voters do not participate in the election, there are four conditions in votes: assent, opposability, neutrality and abstention, then it is an incomplete factor space [2].

Intuition Characteristic Based on Factor Perceptions. Factor perception process is so ordinary that we cannot give an exact definition. It is the focus of study objects; its meaning is found expression in intuition characteristic selection of objects, such as temperature, size and so on. Meanwhile we assume the factor perception field is presented accordingly when a perception process of study objects is pointed out. Consequently any object in the perception field is one-to-one correspondence with the set of its perception process. It includes the following: The judgment of intuition is needed to appoint its perception process. The same object will have different intuition characteristics in different perception process. In fact, the computing model of human brain is an intuitive learning model based on the comparison of optimum perceptions (acceptable perceptions) and non-optimum perceptions (unacceptable perception). [3-7]

Definition2.1[3] Let $F=\left\{f_{1}, f_{2}, \cdots, f_{m}\right\}$ be a set of factors, $A=\left\{A_{1}, A_{2}, \cdots, A_{n}\right\}$ be a set of attributes and $I(A)$ a binary relation defined between $\mu_{O}(A) \in[0,1]$ and $\mu_{\bar{O}}(A) \in[-1,0]$, where $\mu_{\bar{O}}(A)$ be a degree of fuzzy optimum perception for attributes set $A, \mu_{\bar{O}}\left(A_{i}\right)$ be a degree of fuzzy non-optimum perception for attribute $A$, then $I\left(A_{i}\right)=\left\{<\mu_{O}\left(A_{i}\right), \mu_{\bar{O}}\left(A_{i}\right)>\mid \mu_{O}\left(A_{i}\right) \in \mu_{O}(A) \wedge \mu_{\bar{O}}\left(A_{i}\right) \in \mu_{\bar{O}}(A)\right\}$ be called a set of sub-optimum perception of $A$. $I\left(A_{i}\right)$ is also called an intuitive index of factor perception (IIFP), without loss of generality, we have

$$
I\left(A_{i}\right)=\left\{\begin{array}{l}
1 \\
0 \\
\frac{1}{2}\left\{1+\left[\mu_{O}\left(A_{i}\right)-\mu_{\bar{O}}\left(A_{i}\right)\right]\right\} \\
0.5
\end{array}\right.
$$

In the definition 2.1,

$$
\mu_{O}(A)=\left\{\mu_{O}\left(A_{1}\right), \cdots, \mu_{O}\left(A_{n}\right)\right\} \mu_{\bar{O}}(A)=\left\{\mu_{\bar{O}}\left(A_{1}\right), \cdots, \mu_{\bar{O}}\left(A_{n}\right)\right\}(i=1, \cdots, n)
$$

with nature comes to following:

(1) If $I\left(A_{i}\right)=1$, then with maximum certain degree for the perception of the intuition judgment, that is, with maximum fuzzy optimum perception degree for sub-optimum sets.

(2) If $I\left(A_{i}\right)=0$, then with minimum certain degree for the perception of the intuition judgment, that is, with minimum fuzzy optimum perception degree for sub-optimum sets. 
(3) If $0<I\left(A_{i}\right)<1$, then there be an IFPI set as $I\left(A_{i}\right)=\left(I\left(A_{1}\right), \cdots I\left(A_{n}\right)\right)$.

(4) If $I\left(A_{i}\right)=0.5$, then with maximum sub-optimum degree for the fuzzy perception of the intuition judgment.

\section{Applications in Criminal Investigation Reasoning}

In the application of intuitive factor space (IFS) to the collection of Dalian Police Department (DPD) case records, a number of modifications were employed. Provides more detail on the IFS. IFS refer to a general method or criterion in trust intuition discovery. By analysis, we find that IFS is a useful tool in constructing these trusted fuzzy reasoning system [4]. In criminal investigation, the IFS in the knowledge base have forms as follows:

(1)Case-factor(What);(2)Time-factor(When);(3)Scene-factor(Where); (4) Tools-factor (Which); (5) State-factor (How);(6) Reason-factor (Why); (7) Human-factor (Who).

A causal attributes analysis problem was formulated based on the crime dataset from the CIDSS to test the performance of the intuitive factors with multi-perception. The data set contained 150 records, 50 each for three types of kill case, each containing measurements of seven factors (case-factor, time-factor, scene-factor, tools-factor, state-factor, reason-factor and human-factor). The task was to develop an intuition reasoning model from the data, induce rules from these models, and solve attributes analysis problems with the factor perception (see Fig.1) [8-10].

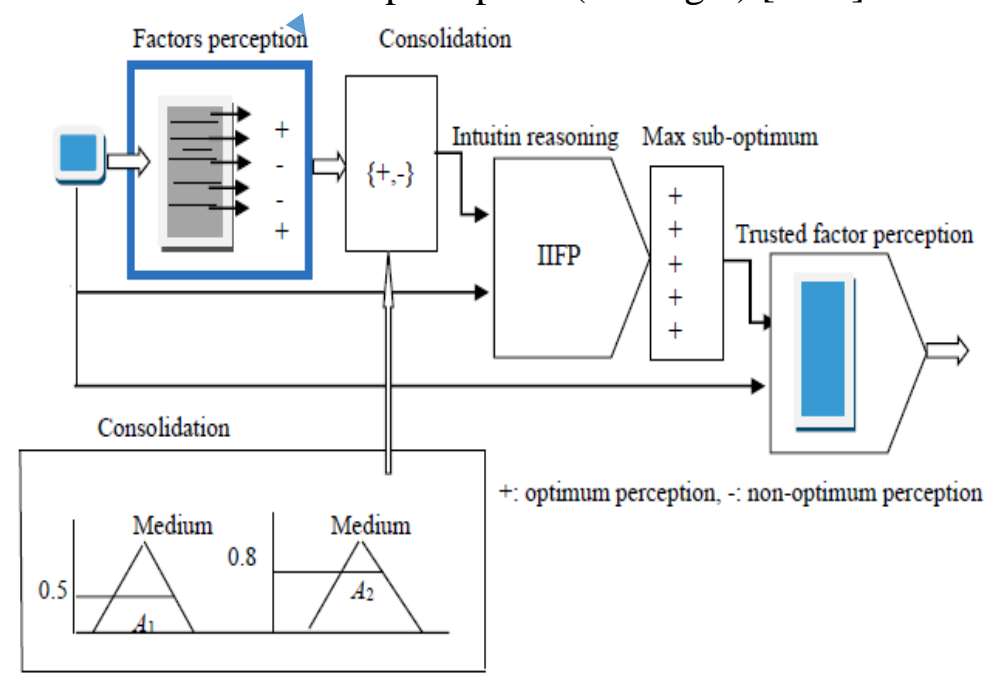

Figure 1. Learning system of factor perception

In the Fig.1, a forward-chaining, factors perception, intuitive reasoning scheme. After factor firing, recurring outputs of the same attribute of an intuitive factor are consolidated so there is only one instance for each attributes of that intuitive factor. The consolidated attribute values of intuitive factor become inputs for the next iteration. For example, in the first iteration two factors perception might fire which result in fuzzy variables, the first giving a fuzzy subset $A_{1}$ with a height of 0.5 in the fuzzy set "Medium" and an associated IIFP1of 0.6, and the second giving a fuzzy subset $A_{2}$ with a height of 0.8 in the fuzzy set "Medium" with IIFP2of 0.7. After consolidation, those two variables will be combined into a single one, with a new fuzzy subset $A$ in "Medium" and an associated IIFP. The iterative process continues until no more factors perception can be fired. According to the key characteristics of intuitive reasoning discussed in intuition learning system. Numerical values of the seven factors mapped onto five fuzzy subsets, i.e., Very Low perception, Low perception, Medium perception, High perception, Very High perception, or five "classes", for each factors. Each numerical value then was represented by the fuzzy subset (class) in which it had the highest membership. For the sake of simplicity, the operation of fuzzy variables was not implemented in this experiment. The variables representing the seven factors, after their values being mapped into classes, were considered ordinal variables and the case type variable was considered a categorical variable [1]. 
The sub-optimum model is a description of the characteristics of human perception which are to be mimicked in sub-optimum learning. Fuzzy set were chosen as the format for perception representation in this study because they are explicit and are easy to implement ((Zadeh 1989). Many researchers have employed fuzzy set theory to construct models of human choice with optimum perception and non-optimum. A sub-optimum learning is comprised of a set of model that are tested (premise), and a perception action that is performed when the premise is satisfied (consequent). In this study, the perception available in sub-optimum learning is embodied in a set of rules and the highly branched learning process is performed by an inference algorithm which operates on the basis of the fuzzy set. Note that the sub-optimum model does not include details of implementation, since those are optimum perception and non-optimum perception.

\section{Conclusions}

This paper discusses the critical issues in establishment of crime investigation reasoning system that should be paid attention to through practice of criminal investigation work. The development of the crime reasoning system must be grounded on factor space, otherwise this work is of little significance or value. Simultaneously, the factor perception should be distinguished from experiential reasoning. For different cases, intuitive reasoning is variable. Only by combining the two together with intuition to reach cooperative reasoning can they possibly play their roles in reality. Besides, the operation mechanism of the trusted fuzzy computing system should apply the IIFP principle, a very useful intellectual system, which is certain to play a guiding role in the development of automatic reasoning computer. Because of the limitation of length of this paper, the detailed procedure is not given and those who are interested can contact the author for more information.

\section{Acknowledgements}

The authors are grateful for the support given by National Natural Science Foundation of China (Grant No. 61272170). We also thank reviewers for insightful and helpful suggestions.

\section{References}

[1] L.A. Zadeh, A new direction in AI: toward a computational theory of perceptions, AI Magazine, 2001, Vol.22, pp.73-84.

[2] Wang Perzhuang, Factor spaces and factor data-bases, Journal of Liaoning Technical University (Natural Science), 2013, Vol.32, pp.1297-1304.

[3] Atanassov K. Intuitionistic fuzzy sets. Fuzzy Set and System, 1986, Vol.20, pp.87-96.

[4] He Ping, Crime Knowledge Management Based on Intuition Learning System, Fuzzy System and Management Discovery, In:Jun Ma, ed, proc. of the Int'l conf IEEE Computer Society, 2008, pp. 555-559.

[5] Weidong Tao, Ping He, Kaiqi Zou. An Approach of Trusted Fuzzy Computing Based on intuition Inversion Neural Network, ICIC Express Letters, Part B: Applications, 2015, pp.1929-1936.

[6] He Ping, Tao Weidong, A Design of Criminal Investigation Expert System Based on CILS, Journal of Software, 2011, Vol.6, No.8, pp1586-1592.

[7] Yung-Chien Sun, Grant Clark.A Computational Model of an Intuitive Reasoner for Ecosystem Control. Expert Systems with Applications, 2009, Vol.36, pp. 12529-12536.

[8] Sun Y.-C. and Clark O. G. Implementing an Intuitive Reasoner with a Large Weather Database. In Proceedings of the World Academy of Science, Engineering and Technology, 2009, vol.50, pp. 500-517. 